\title{
PRÁTICAS DE SUSTENTABILIDADE, GOVERNANÇA CORPORATIVA E RESPONSABILIDADE SOCIAL AFETAM O RISCO E O RETORNO DOS INVESTIMENTOS?
}

\author{
DOES SUSTAINABILITY PRACTICES, \\ CORPORATE GOVERNANCE AND SOCIAL \\ RESPONSIBILITY AFFECT RISK \\ AND RETURN OF INVESTMENTS?
}

Recebido 25/10/2012 Aceito $21 / 01 / 2013$

Bruno Milani ${ }^{1}$ Marcelo Brutti Righi Paulo Sérgio Ceretta ${ }^{3}$ Valéria da Veiga Dias ${ }^{4}$

\section{RESUMO}

O presente artigo tem como objetivo verificar se os investimentos em empresas com melhores práticas de Responsabilidade Social Empresarial, Governança Corporativa e Sustentabilidade apresentam diferenças quanto à performance em relação a investimentos nas empresas que representam o mercado. Para tanto, foram analisadas as séries de retornos diários dos índices Ibovespa, IGC, IGCT, ISE e ITAG através do modelo GARCH e de testes não paramétricos de diferença de média. Os resultados demonstram que a volatilidade condicional dos índices de práticas diferenciadas é significativamente menor do que a volatilidade do índice Ibovespa apesar da correlação entre os retornos ser muito alta. Além disso, o Índice de Sharpe (1966) demonstrou que o retorno por unidade de risco é significativamente superior para os índices de melhores práticas, em relação ao Ibovespa. Assim, de uma maneira geral, é possível concluir que os investimentos em empresas com melhores práticas constituem uma alternativa menos arriscada e mais rentável para o investidor.

Palavras-chave: Performance; Sustentabilidade; Governança Corporativa; Responsabilidade Social

\footnotetext{
${ }^{1}$ Doutorando em Administração pelo Progama de Pós Graduação em Administração da Universidade Federal de Santa Maria (PPGA-UFSM). Professor da Faculdade Palotina de Santa Maria (FAPAS). Santa Maria, Rio Grande do Sul, Brasil. E-mail: milani_ bruno@yahoo.com.br

${ }^{2}$ Mestrando em Administração do Programa de Pós-Graduação em Administração da Universidade Federal de Santa Maria (PPGA-UFSM). Santa Maria, Rio Grande do Sul, Brasil. E-mail: marcelobrutti@hotmail.com

${ }^{3}$ Doutor em Engenharia da Produção pela Universidade Federal de Santa Catarina (UFSC). Professor do Departamento de Ciências Administrativas da Universidade Federal de Santa Maria (UFSM). Santa Maria, Rio Grande do Sul, Brasil. E-mail: ceretta@ smail.ufsm.br

${ }^{4}$ Mestra em Administração pelo Programa de Pós Graduação em Administração da Universidade Federal de Santa Maria (PPGA-UFSM). Professora da Faculdade Palotina de Santa Maria. Santa Maria, Rio Grande do Sul, Brasil. E-mail: aleria-adm@hotmail.com
} 


\section{ABSTRACT}

This article aims to verify whether the investments in companies with better practices of Corporate Social Responsibility, Corporate Governance and Sustainability presents performance differences in relation to investments in companies that represent the market. To this end, we analyzed the series of daily returns of Ibovespa, IGC, IGCT, ISE and ITAG through GARCH model and mean differences tests. The results show that the conditional volatility of the differentiated practices indexes is significantly smaller than the Ibovespa volatility, although the correlation between the returns is very high. Besides that, the Sharpe Index (1966) of the better practices indexes shows that their return per unit of risk is significantly higher than Ibovespa's. Thus, in general, it is possible to conclude that investments in different practices constitute a less risky and more profitable alternative to the investor.

Keywords: Performance; Sustainability; Corporate Governance; Social Responsability

\section{INTRODUÇÃO}

As práticas de Sustentabilidade, Governança Corporativa e Responsabilidade Social Empresarial têm sido tema de múltiplos estudos, tendo como principal origem o trabalho de Bowen (1957), o qual propôs uma visão de Responsabilidade Social alinhada com as políticas da empresa, em contraponto a abordagem filantrópica que o tema carregava nos primórdios.

Nesse sentido, um dos principais questionamentos vigentes diz respeito ao retorno financeiro proporcionado pelos investimentos de empresas que lastreiam suas políticas nessas melhores práticas. Seriam os investimentos responsáveis apenas mais um custo pra empresa? Sem dúvida, as melhores práticas podem ser benéficas para os stakeholders, mas os shareholders são também recompensados?

A BMF\&BOVESPA desenvolveu diversos índices para mensurar o desempenho de empresas que adotam práticas diferenciadas de gestão, ou seja, práticas de Sustentabilidade, Governança Corporativa e Responsabilidade Social. Assim, o retorno financeiro das empresas com práticas diferenciadas pode ser calculado separadamente do retorno das empresas que representam o mercado de maneira global, ou seja, das empresas listadas no Ibovespa.

Os índices que visam mensurar as práticas diferenciadas são: Índice Carbono Eficiente (ICO-2), Índice de Ações com Governança Corporativa Diferenciada (IGC), Índice de Ações com Governança Corporativa Diferenciada Trade (IGCT) e Índice de Ações com Tag Along Diferenciado (ITAG). O índice Ibovespa tradicionalmente é utilizado como proxy de mercado.

Assim, o objetivo deste estudo é comparar a performance dos índices de melhores práticas com a performance do índice Ibovespa, que representa o mercado ordinariamente, ou seja, sem distinções. Para tanto, as análises serão baseadas na lógica do risco/retorno, proposta por Markowitz (1952), utilizando-se como ferramenta o Índice de Sharpe (1966). Contudo, em vez de tomar como medida de risco o desvio-padrão, conforme proposto por Sharpe (1966), será calculada volatilidade condicional através de um modelo $\mathrm{GARCH}$, de acordo com o proposto por Engle (1982) e Bollerslev (1986). Por fim, para verificar se o retorno por unidade de risco difere entre os diferentes índices, será efetuado um teste não paramétrico de diferença de média.

\section{AS PRÁTICAS DE SUSTENTABILIDADE, GOVERNANÇA E RESPONSABILIDADE SOCIAL}

Os estudos publicados acerca de Responsabilidade Social Empresarial (RSE), também conhecida como Responsabilidade Social Corporativa (RSC), têm uma história de cerca de cin- 
qüenta anos, sendo as primeiras décadas dedicadas a construir definições para o termo (CARROLL, 1999). Mesmo com muitos estudos realizados, ainda existem inúmeras definições que relacionam responsabilidade social corporativa, responsabilidade socioambiental, entre outros. Alguns conceitos incluem questões ambientais, outros apenas fatores sociais, ainda existem linhas que trabalham aspectos diversos, como social econômico e ambiental, etc., surgindo também conceitos como sustentabilidade, que, em alguns casos, significa uma consciência social ou socioambiental; ou seja, cada conceito foi criado baseado em uma análise e um ambiente, justificando tantas formas de entender e conceituar o tema.

Em seus primórdios, a responsabilidade social ainda era concebida como caridade, atividades filantrópicas, puramente de auxilio comunitário ou social, mas Bowen (1957) realizou um dos estudos mais significativos sobre RSC, já que foi o primeiro autor a propor uma nova visão da mesma, que deixaria de ser apenas um conjunto de ações paternalistas de filantropia para estar inserida nas políticas das organizações.

Leff (2001) destaca que, desde o início das atividades industriais, o ambiente não poderia ser considerado apenas como uma realidade visível, mas sim uma convergência de processos físicos, biológicos e simbólicos, que, por meio das ações econômicas, científicas e técnicas do homem, são reorganizados e reconduzidos; e muitos países já instalaram um processo de educação ambiental e social que caminha nesse sentido. No Brasil, embora o tema esteja em discussão desde o início da década de 90, a inserção do assunto dentro da rotina das organizações é gradual.

A partir dessas transformações no ambiente e na sociedade, novos conceitos foram considerados dentro do cenário organizacional, tais como Responsabilidade Social Empresarial (Corporativa), Governança Corporativa, Gestão Ambiental, Sustentabilidade e Desenvolvimento Sustentável. Mesmo considerando que possa haver diferentes fins para esses conceitos, no geral, todos buscam uma atitude de consciência moral para com os stakeholders e o ambiente. Quando se trata desses conceitos, ainda há uma falta de consenso por parte dos teóricos e estudiosos a respeito das definições corretas do termo sustentabilidade Uma forma generalizada de definição no âmbito de mercado é que refere às atividades que evidenciam a inclusão de aspectos sociais e ambientais nas atividades fins e nas interações com stakeholders. O Quadro 1 visa a distinguir esses conceitos.

Quadro 1 - Comparativos dos Conceitos Atuais

\begin{tabular}{|l|l|l|l|l|l|}
\hline $\begin{array}{l}\text { Critério/Con- } \\
\text { ceito }\end{array}$ & $\begin{array}{l}\text { Responsabili-dade } \\
\text { Social Empresarial } \\
\text { (RSE) }\end{array}$ & Ética nos \\
negócios (EN) & $\begin{array}{l}\text { Gerenciamento de } \\
\text { Stakeholders (GS) }\end{array}$ & $\begin{array}{l}\text { Sustentabilidade } \\
\text { (SUS) }\end{array}$ & $\begin{array}{l}\text { Cidadania } \\
\text { Empresarial (CE) }\end{array}$ \\
\hline História & 1960(e anterior) & 1970 & 1980 & 1990 & 2000 \\
\hline Origem & Impacto Social & Moralidade & $\begin{array}{l}\text { Não acionistas } \\
\text { (sócios) }\end{array}$ & Meio Ambiente & $\begin{array}{l}\text { Comunidade /Filan- } \\
\text { tropia }\end{array}$ \\
\hline $\begin{array}{l}\text { Principal } \\
\text { definição }\end{array}$ & Fazer o bem & Evitar danos & $\begin{array}{l}\text { Equilibrar de Inte- } \\
\text { resses }\end{array}$ & $\begin{array}{l}\text { Preservação do } \\
\text { futuro }\end{array}$ & Contribuição social \\
\hline Entidade foco & Organizações & $\begin{array}{l}\text { Gestores, colabo- } \\
\text { radores e organi- } \\
\text { zações }\end{array}$ & $\begin{array}{l}\text { Gestores e organi- } \\
\text { zações }\end{array}$ & Organizações & Organizações \\
\hline $\begin{array}{l}\text { Versões limita- } \\
\text { das VS. Amplas }\end{array}$ & $\begin{array}{l}\text { Econômico/legal } \\
\text { VS. Ética discricio- } \\
\text { nária }\end{array}$ & $\begin{array}{l}\text { Direito / cumpri- } \\
\text { mento VS ética/ in- } \\
\text { tegridade e valores }\end{array}$ & $\begin{array}{l}\text { Limitado/ primário } \\
\text { e stakeholder VS. } \\
\text { Amplo secundário } \\
\text { e stakeholder }\end{array}$ & $\begin{array}{l}\text { Homocêntrica VS. } \\
\text { Ecocêntrica }\end{array}$ & $\begin{array}{l}\text { Filantropia/ relação } \\
\text { com comunidade } \\
\text { VS. Social/ global }\end{array}$ \\
\hline
\end{tabular}




\begin{tabular}{|c|c|c|c|c|c|}
\hline $\begin{array}{l}\text { Critério/Con- } \\
\text { ceito }\end{array}$ & $\begin{array}{l}\text { Responsabili-dade } \\
\text { Social Empresarial } \\
\text { (RSE) }\end{array}$ & $\begin{array}{l}\text { Ética nos } \\
\text { negócios (EN) }\end{array}$ & $\begin{array}{l}\text { Gerenciamento de } \\
\text { Stakeholders (GS) }\end{array}$ & $\begin{array}{l}\text { Sustentabilidade } \\
\text { (SUS) }\end{array}$ & $\begin{array}{l}\text { Cidadania } \\
\text { Empresarial (CE) }\end{array}$ \\
\hline $\begin{array}{l}\text { Ramificações } \\
\text { teóricas }\end{array}$ & \begin{tabular}{|l|} 
Modelo sharehol- \\
der \\
Responsabilidade \\
empresarial e so- \\
cial empresarial \\
Questões sociais e \\
gerenciamento \\
Performance social \\
empresarial \\
\end{tabular} & $\begin{array}{l}\text { Ética baseada em } \\
\text { gerenciamento } \\
\text { Valores baseados } \\
\text { em gerenciamento } \\
\text { Governança corpo- } \\
\text { rativa e ética } \\
\text { Relacionamento } \\
\text { moral e ético }\end{array}$ & \begin{tabular}{|l|} 
Relação com \\
stakeholder \\
Comprometimen- \\
to com stakehol- \\
der \\
Sociedade e stake- \\
holder \\
Capitalismo e \\
stakeholder \\
\end{tabular} & $\begin{array}{l}\text { Desenvolvimento } \\
\text { empresarial } \\
\text { Negócios e o de- } \\
\text { senvolvimento } \\
\text { Capitalismo sus- } \\
\text { tentável }\end{array}$ & $\begin{array}{l}\text { Negócios e cida- } \\
\text { dania } \\
\text { Cidadania e negó- } \\
\text { cios globais }\end{array}$ \\
\hline $\begin{array}{l}\text { Razão } \\
\text { (justificativa } \\
\text { teórica) }\end{array}$ & $\begin{array}{l}\text { Contrato social } \\
\text { Agencia moral } \\
\text { Poder social } \\
\text { Interpenetração } \\
\text { Stakeholder } \\
\text { Utilitarista } \\
\text { Bases e proprie- } \\
\text { dades } \\
\text { Religião }\end{array}$ & $\begin{array}{l}\text { Utilitarismo } \\
\text { Deontologia } \\
\text { Direitos morais } \\
\text { Justiça } \\
\text { Virtudes morais }\end{array}$ & $\begin{array}{l}\text { Agencia } \\
\text { Justiça } \\
\text { Contrato social } \\
\text { Direitos morais } \\
\text { Kantismo } \\
\text { Utilitarismo } \\
\text { Liberalismo } \\
\text { Confiança } \\
\text { Ética feminina }\end{array}$ & $\begin{array}{l}\text { Utilitarismo } \\
\text { Direitos morais }\end{array}$ & $\begin{array}{l}\text { Utilitarismo } \\
\text { Contrato social } \\
\text { Direitos morais }\end{array}$ \\
\hline
\end{tabular}

Fonte: (Elaborado a partir de SCHWARTZ e CARROLL, 2008 apud Dias, 2011)

De acordo com Schwartz e Carroll (2008) quando se trata da relação entre negócios e sociedade, surge muita competição por proeminência nos assuntos concernentes a responsabilidade social, ambiental ou desenvolvimento sustentável. Embora discutível, os contendores primário parecem incluir (a) responsabilidade social empresarial ou corporativa (RSE/ RSC), (b) ética dos negócios (EN), (c) gerência de stakeholders (GS), (d) sustentabilidade (SUS), e (e) cidadania corporativa (CC). Há pouco tempo, ainda pairavam muitas dúvidas a respeito da legitimidade teórica ou prática desses conceitos; no entanto, essa situação parece ter mudado consideravelmente, dada a importância conquistada pelo tema dentro do contexto global, considerando a inclusão no meio social, ambiental ou mesmo o empresarial.

Apesar da prevalência dos cinco conceitos diversos, e de certa forma consolidados em suas abordagens, ainda existem algumas dificuldades na compreensão do que cada um realmente significa, ou melhor, no modo como cada um pode se relacionar com os outros. O resultado é certo grau de confusão na compreensão da sociedade e até mesmo do meio acadêmico, o que prejudica a consolidação do campo e o desenvolvimento teórico.

A prova da confusão entre os construtos é facilmente perceptível, já que cada um dos conceitos tem sido sugerido para incorporar um ou mais dos outros de acordo com diversos autores. Conforme Werre e Marrewijk (2003 p. 107) apud Schwartz e Carroll (2008), equipara-se sustentabilidade com a RSE: "sustentabilidade corporativa, e também a RSE, refere-se às atividades de uma empresa-voluntário por definição". Willard (2002 p. 6) equivale sustentabilidade com a RSE e CC e afirma "o desenvolvimento sustentável, também conhecido como a responsabilidade social das empresas, boa cidadania corporativa".

Werre e Marrewijk (2003, p. 95-96) resumem: "um intenso debate tem ocorrido entre os acadêmicos, consultores, e executivos de empresas, resultando em muitas definições de uma forma mais humana, mais ética e uma forma mais transparente de fazer negócios". Criaram-se conceitos relacionados, tais como desenvolvimento sustentável, cidadania corporativa, Triple Bottom Line, ética empresarial, e responsabilidade social corporativa [...] uma variedade de conceitos, definições colocou os executivos de negócios em uma situação embaraçosa, especialmente aqueles que estão começando a assumir a sua responsabilidade perante a sociedade e 
os seus intervenientes, deixando-os com mais perguntas do que respostas. Para ajudar a resolver a confusão, vários pesquisadores têm tentado analisar e avaliar cada constructo.

\section{ESTUDOS ACERCA DA PERFORMANCE E VOLATILI- DADE DE EMPRESAS COM PRÁTICAS DIFERENCIADAS}

Ortas, Moneva e Salvador (2010) estudam a volatilidade dos índices da bolsa de Madrid, comparando índices tradicionais de mercado com índices associados à sustentabilidade. O mercado espanhol é representado pelo IBEX, e o mercado de empresas associadas a práticas sustentáveis é representado pelo índice FTSE4GOOD-IBEX, um integrante da família FTSE que visa a mensurar o desempenho das empresas com boas práticas, dentro do universo do IBEX. Baseando-se numa amostra de dados de frequência diária de 09/04/2008 a 05/02/2010, inicialmente, estima-se o CAPM, que resulta em Alfa significativamente negativo e Beta aproximadamente igual a um. Em seguida, a volatilidade é estimada com base nos modelos GARCH, GJR e EGARCH, os quais demonstram que a volatilidade do índice FTSE4GOOD-IBEX é inferior a do IBEX, resultado que é confirmado por modelos multivariados. Ainda, destaca-se que os modelos assimétricos geram coeficientes de assimetria positivos e significativos, demonstrando que o impacto de choques negativos na volatilidade é maior do que choques positivos.

Hoti, McAleer e Pauwels (2008) analisam as diferenças entre a volatilidade dos índices DJIA e S\&P500 e os índices DJSI e ESI, sendo que os primeiros representam mercados como um todo, e os demais são indicadores de sustentabilidade. Com dados de frequência diária de 31/12/1997 a 01/09/2005, as volatilidades e covariâncias das séries são estimadas através do modelo VARMA-GARCH. Seus resultados demonstram que o DJSI é afetado pelos seus coeficientes Alfa ( $\alpha$ ) e Beta ( $B)$, os quais representam, respectivamente, os choques de curto e longo prazo, além dos coeficientes $\alpha$ e $B$ dos índices ESI e DJIA. Por outro lado, o ESI é afetado apenas pelo seu próprio $B$.

A diferença poderia ser explicada pelo critério de composição do índice: o DJIA é composto excluindo as ações que não se encaixam nos seus critérios de sustentabilidade, ou seja, ações de empresas envolvidas em atividades controversas; o ESI é composto pelas ações de empresas que demonstram atitude pró-ativa para melhores práticas de sustentabilidade. Ou seja, um índice utiliza um critério positivo e o outro, negativo.

Assim, Hoti, McAleer e Pauwels (2008) verificam que há spillover de volatilidade do ESI para o DJIA, para o DJSI e para o S\&P500, mas não o contrário. Contudo, choques de volatilidade no DJIA afetam o DJSI. Dessa forma, concluem que choques nos índices de volatilidade afetam os índices de mercado, mas não o contrário, exceto para o último caso. Deve ser levado em conta que o ESI é muito mais restritivo do que o DJSI, o que explica porque sua volatilidade é menos afetada pelo mercado. Contudo, de maneira geral, as diferenças não foram significativas o suficiente para afirmar que os índices não estão sujeitos ao mesmo nível de risco.

Jubert et.al. (2008) estudam o padrão de volatilidade dos principais índices da Bovespa, entre eles, o Índice de Sustentabilidade empresarial (ISE), analisando dados de frequência diária do período 2006-2007, através de vários modelos da família ARCH. Seus resultados demonstram que o ISE apresenta o menor valor de $\alpha$ entre os índices estudados, ou seja, as amplitudes das variações dos preços das ações do ISE reagem, inicialmente, com um movimento menor do que os demais índices. Contudo, o coeficiente $\beta$ do ISE é o segundo maior, indicando que a volatilidade do dia anterior é transmitida para o dia seguinte. De uma maneira geral, o índice Ibovespa foi considerado o menos volátil, resultado atribuído ao seu alto grau de diversificação, assim como o 
ITEL foi considerado o mais volátil, seguido do ISE.

Rabelo et. al. (2007) estudam a performance de melhores práticas de governança corporativa no Brasil, com base nos CAPM e em diversos índices, como Treynor, Sharpe e Sortino. Os autores definem dois tipos de carteiras de investimento: um de empresas que apresentam práticas de governança corporativa superior (GCD) e outra de empresas que não as apresentam (GNCD), excluindo empresas que faziam parte do IGC e do lbovespa simultaneamente e empresas financeiras. Os critérios para a formação das carteiras de empresas que apresentam boas práticas foram a presença no IGC e no Novo Mercado da Bovespa. Para igualar o tamanho das carteiras, foram excluídas da lista de empresas GNCD as menos líquidas. Com base em dados de 2006 e numa estimação bootstrap, que permitiu a formação de inúmeras carteiras, os resultados apontam que as carteiras GCD apresentam risco e retorno superiores, sendo que a aplicação dos índices que ajustam o retorno pelo risco apontou que as carteiras GCD têm também a melhor relação risco/retorno.

Sarianiddis et. al. (2009) estudam o efeito do impacto de variáveis macroeconômicas sobre a volatilidade dos índices DJSI e DJW, no período de fevereiro de 1999 a janeiro de 2008, com dados de frequência mensal. Com base nos modelos GARCH e IGARCH multivariados, seus resultados demonstram que diversas variáveis macroeconômicas, como taxas de juros, preço do petróleo, câmbio e desemprego exercem influência sobre a volatilidade dos índices; mas, no caso do DJSI, índice que representa as empresas consideradas sustentáveis, os choques de volatilidade são mais lentamente assimilados, pois não apresenta $\alpha$ significativo, ao passo que seu $\beta$ é significativo e superior ao do índice DJW.

\section{4 ÎNDICES DE MERCADO E DE SUSTENTABILIDADE BRASILEIROS}

O Índice Ibovespa, segundo Oliveira e Pacheco (2010), é o mais importante indicador do desempenho médio das cotações do mercado de ações brasileiro. Sua relevância advém do fato do Ibovespa retratar o comportamento dos principais papéis negociados na BM\&FBOVESPA. 0 critério para a inclusão de ações é estar incluída em uma relação cujos índices de negociabilidade representem $80 \%$ do valor acumulado de todos os índices individuais. O Ibovespa é comumente utilizado como benchmark do mercado brasileiro.

O IBrX-100, segundo a BM\&FBOVESPA (2012), é um índice que mede o retorno total de uma carteira teórica composta por 100 ações selecionadas entre as mais negociadas na BM\&FBOVESPA em termos de liquidez. Oliveira e Pacheco (2010) ressaltam que esse índice considera apenas as ações que não estão sob posse do controlador da empresa, tendo sido criado em 1997. Posteriormente, a Bovespa criou o IBrX-50, com os mesmos objetivos e critérios, exceto pelo fato de que considera apenas as cinqüenta ações mais líquidas.

Com o intuito de valorizar boas práticas referentes à sustentabilidade, responsabilidade social e governança corporativa, a BM\&FBOVESPA criou diversos índices que visam a mensurar o retorno de ações de empresas com práticas diferenciadas. Nesse sentido, o ISE foi criado como um esforço conjunto da BM\&FBOVESPA, com várias instituições, como a Associação Brasileira das Entidades Fechadas e de Previdência Privada (ABRAPP), a Associação Brasileira das Entidades dos Mercados Financeiros e de Capitais (ANBIMA), a Associação dos Analistas e Profissionais de Investimento do Mercado de Capitais (APIMEC), o Instituto Brasileiro de Governança Corporativa (IBGC), o Instituto de Fiscalização e Controle (IFC), o Instituto ETHOS e o Ministério do Meio Ambiente (BM\&FBOVESPA, 2012), os quais formam um conselho deliberativo responsável por um 
questionário que avalia as práticas de sustentabilidade de cada empresa e do impacto dos produtos e serviços para a sociedade (FORTUNA, 2008, p. 627). Seu conceito-base é o de triple bottom line, que avalia elementos econômico-financeiros, sociais e ambientais de forma integrada. O ISE engloba até quarenta empresas, que se aproximam da excelência na gestão da sustentabilidade.

O Índice Carbono Eficiente (ICO-2) foi criado com base nas preocupações com o aquecimento global. É formado por empresas participantes do IBrX-50 que aceitaram ser avaliadas pelo seu grau de eficiência nas emissões de geradores do efeito estufa (BM\&FBOVESPA, 2012)

O Índice de Ações com Governança Corporativa Diferenciada (IGC) visa a compor uma carteira de empresas com bom nível de governança corporativa. Para fazer parte do IGC, as empresas devem ser negociadas no Novo Mercado ou classificadas nos níveis 1 e 2 da BM\&FBOVESPA, conforme Fortuna (2008). Com base no IGC, foi criado o IGC Trade ou, simplesmente, IGCT, que trata de uma relação de ações cujos índices de negociabilidade somados representem $99 \%$ do valor acumulado de todos os índices individuais (BM\&FBOVESPA, 2012). A diferença entre o IGC e o IGCT, basicamente, é que o IGCT fixa critérios de liquidez para a composição do índice.

O ITAG - Índice de Ações com Tag Along Diferenciado - tem por objetivo, conforme Oliveira e Pacheco (2010), medir o desempenho de uma carteira teórica composta por ações de empresas que ofereçam melhores condições aos acionistas minoritários no caso de alienação do controle. Elegem-se à inclusão na carteira teórica do índice todas as ações para as quais a empresa concede tag along superior em relação à legislação aplicável, a qual estabelece que as companhias abertas devem oferecer a todas as ações ordinárias minoritárias tag along de $80 \%$ em relação ao preço obtido pelo controlador, no caso de alienação de controle. Dessa forma, as ações ordinárias cujas companhias oferecem um percentual maior de tag along e/ou as ações preferenciais cuja companhia oferece tag along em qualquer percentual são elegíveis ao ITAG (BM\&FBOVESPA, 2012).

\section{MÉTODO}

Neste estudo, analisam-se os índices Ibovespa, IGC, IGCT, ISE e ITAG, cujas séries de cotações foram obtidas junto ao site da BM\&FBOVESPA e referem-se ao período de 03/01/2006 a 30/03/2012. A frequência é diária, totalizando 1543 observações. O índice ICO-2 não é analisado porque teve início apenas em 01/09/2010. Inicialmente, são analisadas a correlação, as estatísticas descritivas e a normalidade dos log-retornos da amostra.

Em seguida, inicia-se a análise da volatilidade dos índices, conforme metodologia apresentada por Engle (1982) e Bollerslev (1986). Os modelos autoregressivos de heteroscedasticidade condicional surgiram para viabilizar a análise de momentos condicionais superiores, tendo em vista que, anteriormente a esses estudos, o risco era analisado apenas pela variância. Dependências temporais de ordem superior eram perturbações aleatórias, consideradas em seus momentos condicionais. Essas dependências expressam a existência de aglomerações na série e a alternância de períodos de alta volatilidade e de baixa volatilidade (BUENO, 2011, p. 233).

Tendo em vista a importância do risco na teoria econômica moderna, a modelagem temporal de variâncias e covariâncias tornou-se fundamental. Os modelos de heteroscedasticidade condicional (GARCH) fundamentam-se na equação da variância condicional, em vez de considerá-la constante ao longo do tempo.

Para corretamente modelar o comportamento da média e variância condicional das variáveis, optou-se pelo emprego de uma combinação dos modelos auto-regressivos com médias móveis (ARMA) e o já mencionado GARCH. O modelo ARCH de Engle (1982) é um processo esto- 
cástico condicional à informação em t-1. O modelo GARCH de Bollerslev (1986) é uma generalização do ARCH. Tal modelo estimado é exposto conforme Equações [1], [2] e [3]:

$$
\begin{aligned}
& r_{i, t}=\mu_{i}+\sum \phi_{i, m} r_{i, t-m}+\sum \theta_{i, n} \varepsilon_{i, t-n}+\varepsilon_{i, t}, \\
& \varepsilon_{i, t}=h_{i, t} z_{i, t}, z_{i, t} \sim t_{v}, \\
& h_{i, t}=\omega_{i}+\sum \alpha_{i, p} \varepsilon_{i, t-p}^{2}+\sum \beta_{i, q} h_{i, t-q} .
\end{aligned}
$$

Onde $r_{i, t}$ é o log-retorno de um ativo $i$ no período $t ; h_{i, t}$ é a variância condicional de um ativo $i$ no período $t ; \mu_{i}, \phi_{i}, \theta_{i}, \omega_{i}, \alpha_{i}$ e $\beta_{i}$ são parâmetros; $\varepsilon_{i, t}$ é a inovação da média condicional do ativo $i$ no período $t ; z_{i, t}$ representa um ruído branco com distribuição de student com $v$ graus de liberdade. A escolha desta distribuição se justifica por captar bem o conhecido comportamento leptocúrtico de ativos financeiros (LOGIN e SOLNICK, 2001). Para escolher as defasagens que compõe cada modelo, foi utilizado o critério de Akaike (AIC).

Em relação a um tradicional modelo $A R C H$, o modelo $\mathrm{GARCH}$ difere por inserir o coeficiente Beta, que é um vetor autoregressivo da variância condicional. No modelo GARCH, é possível interpretar que o coeficiente alfa representa a variação do erro quadrático no período anterior (efeito $\mathrm{ARCH}$ ) e o coeficiente Beta, a persistência de longo prazo da variância condicional (efeito GARCH). Os modelos serão validados por meio de testes $Q$ de Ljung e Box (1978) nos seus resíduos e resíduos quadrados.

O retorno por unidade de risco será utilizado para mensurar a performance dos índices, conforme Sharpe (1966). A Equação [4] apresenta o índice de Sharpe (1966):

$$
S=\frac{E[R]}{\sigma}
$$

Onde: $E[R]$ é a expectativa de retorno (média); $\sigma$ é o desvio-padrão da série.

Verifica-se que o desvio-padrão é a medida de risco proposta por Sharpe (1966), o que pode ser muito impreciso, principalmente se a variância for heteroscedástica. Para resolver este problema, este estudo utiliza a volatilidade condicional como medida de risco, calculada através de um modelo GARCH, conforme descrito anteriormente.

Além disso, Sharpe (1966) previa que o retorno médio da série deveria ser utilizado, o que não seria adequado neste caso, pois o estudo baseia-se justamente na modelagem temporal da variância. Assim, o índice de Sharpe (1966) foi calculado para cada observação, valendo-se do retorno do período e sua respectiva volatilidade estimada, procedimento repetido para todos os índices estudados. Portanto, o retorno por unidade de risco, tomando como risco a volatilidade condicional, pode ser expresso pela Equação [5]:

$$
S=\frac{R_{t}}{h_{i, t}}
$$

Onde: $R_{t}$ é o retorno no período $t ; h_{i, t}$ é a volatilidade condicional;

Para comparar as séries das volatilidades condicionais e de Índices de Sharpe (1966) estimadas pelas Equações [3] e [5], será aplicado o teste de soma de postos para diferença de mediana de Wilcoxon (1945), para verificar se há diferenças estatisticamente significativas entre elas, ou seja, se a variância condicional e o índice de Sharpe (1966) realmente diferem entre as 
séries. O teste de Wilcoxon (1945) pode ser representado pelas Equações [6] e [7]:

$$
\begin{aligned}
& z=\frac{w-0,5}{\sigma_{w}} \\
& \sigma_{w}=\sqrt{\frac{N_{r}\left(N_{r}+1\right)\left(2 N_{r}+1\right)}{6}}
\end{aligned}
$$

Onde: $w$ é o valor absoluto da soma dos postos; $\sigma_{w}$ é o seu desvio-padrão; $Z$ é o valor crítico, que deve ser comparado com o tabelado pra verificação do $p$-valor e, consequentemente, rejeitar ou não a hipótese nula. Neste caso, a hipótese nula é que as médias sejam iguais.

Na seção 6 apresentou-se os resultados obtidos, acompanhados de uma breve explanação. Com base nos resultados, na seção 7 traçou-se as considerações finais.

\section{RESULTADOS}

Dando início à interpretação dos resultados, apresenta-se a Tabela 1, com as estatísticas descritivas e o teste de normalidade de Jarque-Bera, a fim de verificar se a distribuição das séries se aproxima de uma normal.

Tabela 1 - Estatísticas Descritivas e Teste de Normalidade

\begin{tabular}{lccccc}
\hline & Média & Mínimo & Máximo & Desvio Padrão & p-valor Jarque-Bera \\
\hline Ibovespa & 0,000425 & $-0,1210$ & 0,1368 & 0,0199 & 0,0000 \\
IGC & 0,000474 & $-0,1094$ & 0,1397 & 0,0184 & 0,0000 \\
IGCT & 0,000485 & $-0,1182$ & 0,1496 & 0,0195 & 0,0000 \\
ISE & 0,000514 & $-0,1036$ & 0,1449 & 0,0187 & 0,0000 \\
ITAG & 0,000466 & $-0,1090$ & 0,1554 & 0,0188 & 0,0000 \\
\hline
\end{tabular}

O teste de normalidade de Jarque-Bera rejeita a hipótese nula de que a distribuição dos dados seja normal para todos os índices, ao grau de significância de 1\%. As estatísticas descritivas demonstram que o índice Ibovespa apresenta o menor valor mínimo $(-0,1210)$ e o menor valor máximo $(0,1368)$. O ISE sustenta o maior valor mínimo $(-0,1036)$ e o ITAG, o maior valor de máximo $(0,1554)$. O Ibovespa ainda apresenta o menor retorno médio $(0,000425)$ e o maior desvio-padrão (0,0199); ao passo que a maior média é a do ISE $(0,000514)$, e o menor desvio-padrão é o do IGC $(0,0184)$.

Tomando-se como base a amplitude e o retorno médio das séries, há indícios de que o Ibovespa tenha o pior desempenho, assim como o desvio-padrão indica que sua volatilidade seja mais alta. Enfim, as estatísticas descritivas apontam que o lbovespa é o índice com menor retorno. Dando continuidade, a Tabela 2 evidencia os coeficientes de correlação dos retornos dos índices estudados.

Tabela 2 - Correlação entre os retornos dos índices

\begin{tabular}{cccccc}
\hline Ibovespa & IGC & IGCT & ISE & ITAG & Variável \\
\hline 1 & 0,9779 & 0,9795 & 0,955 & 0,9569 & Ibovespa \\
& 1 & 0,995 & 0,9605 & 0,9874 & IGC \\
& & 1 & 0,9634 & 0,9802 & IGCT \\
& & & 1 & 0,9643 & ISE \\
\hline
\end{tabular}


Verifica-se que a correlação entre os retornos dos índices é elevada, demonstrando que os retornos do Ibovespa, que representa o mercado, e os outros índices compartilham uma variação linear, em torno de suas médias, de pelo menos $95 \%$. Isso significa que grande parte da variação dos índices se dá na mesma direção e intensidade, o que indica que os seus retornos podem ser muito parecidos.

As Tabelas 3, 4 e 5 apresentam os resultados das estimações da volatilidade condicional para os índices. Com o objetivo de otimizar o espaço, cada tabela agrupa as estimações de até dois índices.

Tabela 3 - Volatilidade condicional do Índice Ibovespa

\begin{tabular}{|c|c|c|c|}
\hline \multicolumn{4}{|c|}{ Ibovespa } \\
\hline & Coeficiente & Desvio-Padrão & $\mathrm{p}$-valor \\
\hline \multicolumn{4}{|c|}{ Equação da média condicional } \\
\hline Const & 0,000982 & 0,000377 & \\
\hline \multicolumn{4}{|c|}{ Equação da variância condicional } \\
\hline Omega & 0,8775 & 0,3750 & 0,0193 \\
\hline Alfa2 & 0,0792 & 0,0372 & 0,0334 \\
\hline Beta & 0,8669 & 0,0339 & 0,0000 \\
\hline
\end{tabular}

Parâmetros da densidade condicional

Graus de Liber-

dade

1,7544

0,0000

AIC: $\quad-826,06$

* Valores estimados com base nas Equações [1], [2] e [3].

Os testes $Q$ não rejeitaram a hipótese nula, em todas as estimações, de que os resíduos (lineares e quadráticos) não são autocorrelacionados, o que significa que os modelos GARCH estimados filtraram a informação (dependência) com o passado das séries. O coeficiente alfa foi significativo com uma defasagem de dois períodos, considerando o grau de significância de $5 \%$; enquanto o Beta pode ser considerado significativo com apenas uma defasagem. Tal situação caracteriza um modelo GARCH $(2,1)$.

Dando sequência à discussão dos resultados, na Tabela 4, apresentam-se as estimações dos coeficientes da variância condicional dos índices IGC e IGCT.

Tabela 4 - Volatilidade condicional dos Índices IGC e IGCT

\begin{tabular}{|c|c|c|c|c|c|c|}
\hline & IGC & & & IGCT & & \\
\hline & Coeficiente & Desvio-Padrão & p-valor & Coeficiente & Desvio-Padrão & p-valor \\
\hline \multicolumn{7}{|c|}{ Equação da média condicional } \\
\hline Const. & 0,001131 & 0,000331 & 0,000600 & 0,001073 & 0,000356 & 0,002500 \\
\hline \multicolumn{7}{|c|}{ Equação da variância condicional } \\
\hline Omega & 0,5838 & 0,2292 & 0,0109 & 0,6366 & 0,2636 & 0,0157 \\
\hline Alfa2 & 0,0853 & 0,0347 & 0,0140 & 0,0853 & 0,0342 & 0,0127 \\
\hline Beta & 0,8692 & 0,0283 & 0,0000 & 0,8742 & 0,0284 & 0,0000 \\
\hline \multicolumn{7}{|c|}{ Parâmetros da densidade condicional } \\
\hline $\begin{array}{c}\text { Graus de Liber- } \\
\text { dade }\end{array}$ & 8,3501 & 1,7578 & 0,0000 & 8,7121 & 1,8599 & 0,0000 \\
\hline AIC: & $-8540,05$ & & & AIC: & $-8337,20$ & \\
\hline
\end{tabular}

* Valores estimados com base nas Equações [1], [2] e [3]. 
Da mesma maneira que a equação estimada para o Ibovespa, os índices IGC e IGCT caracterizam modelos GARCH $(2,1)$, ou seja, os modelos apresentam Alfa significativo com duas defasagens e Beta significativo com uma defasagem. Os coeficientes Alfa e Beta do IGC e do IGCT são mais altos do que os estimados para o Ibovespa, especialmente o Alfa. Isso significa que suas equações têm um poder de explicação um pouco maior do que a do lbovespa, além de que estão mais suscetíveis ao impacto do erro de curto prazo. A Tabela 5 demonstra as estimações da volatilidade condicional para os Índices ITAG e ISE.

Tabela 5 - Volatilidade condicional dos Índices Tag Along e ISE

\begin{tabular}{|c|c|c|c|c|c|c|}
\hline & \multirow{2}{*}{$\begin{array}{c}\text { Tag Along } \\
\text { Coeficiente }\end{array}$} & \multicolumn{5}{|c|}{ ISE } \\
\hline & & Desvio-Padrão & p-valor & Coeficiente & Desvio-Padrão & p-valor \\
\hline \multicolumn{7}{|c|}{ Equação da média condicional } \\
\hline Const. & 0,00108 & 0,00034 & 0,00140 & 0,000971 & 0,000329 & 0,003100 \\
\hline \multicolumn{7}{|c|}{ Equação da variância condicional } \\
\hline Omega & 0,6360 & 0,2347 & 0,0067 & 0,4168 & 0,1894 & 0,0278 \\
\hline Alfa2 & 0,0825 & 0,0350 & 0,0185 & 0,0820 & 0,0364 & 0,0242 \\
\hline Beta & 0,8666 & 0,0271 & 0,0000 & 0,8815 & 0,0279 & 0,0000 \\
\hline \multicolumn{7}{|c|}{ Parâmetros da densidade condicional } \\
\hline $\mathrm{Ni}$ & 9,4280 & 2,1854 & 0,0000 & 9,9586 & 2,3128 & 0,0000 \\
\hline AIC: & $-8468,26$ & & & AIC: & $-8529,95$ & \\
\hline
\end{tabular}

Os índices ITAG e ISE também estão sujeitos a um modelo $\operatorname{GARCH}(2,1)$, tendo em vista que o coeficiente Alfa é significativo com duas defasagens; e o Beta, com uma defasagem. A Tabela 5 chama a atenção para o fato de que o ISE apresenta o maior coeficiente Beta, ou seja, é o índice que está mais sujeito à persistência da volatilidade de longo prazo. O coeficiente Alfa é maior do que o do Ibovespa, mas menor do que o do IGC, IGCT e do ITAG, o que permite interpretar que o ISE está mais sujeito a persistência de longo prazo na volatilidade e menos sujeito à variação do erro do período anterior, em relação aos índices que representam as melhores práticas. Para analisar graficamente a volatilidade, as Figuras 1 e 2 apresentam, respectivamente, a variação dos retornos do lbovespa e a variação dos retornos dos índices de melhores práticas.

As Figuras 1 e 2 deixam claro que as variações dos retornos de todos os índices são muito similares, conforme previsto pela análise da correlação na Tabela 01. Dessa forma, não é possível identificar visual e graficamente nenhum indício de que a variação dos retornos difere entre os índices. Tanto na Figura 1 quanto na Figura 2, fica evidente o aumento da volatilidade durante a crise do subprime, o que reforça a necessidade de modelos de volatilidade condicional, tendo em vista que não é constante. 
Figura 1 - Variação dos retornos do Ibovespa

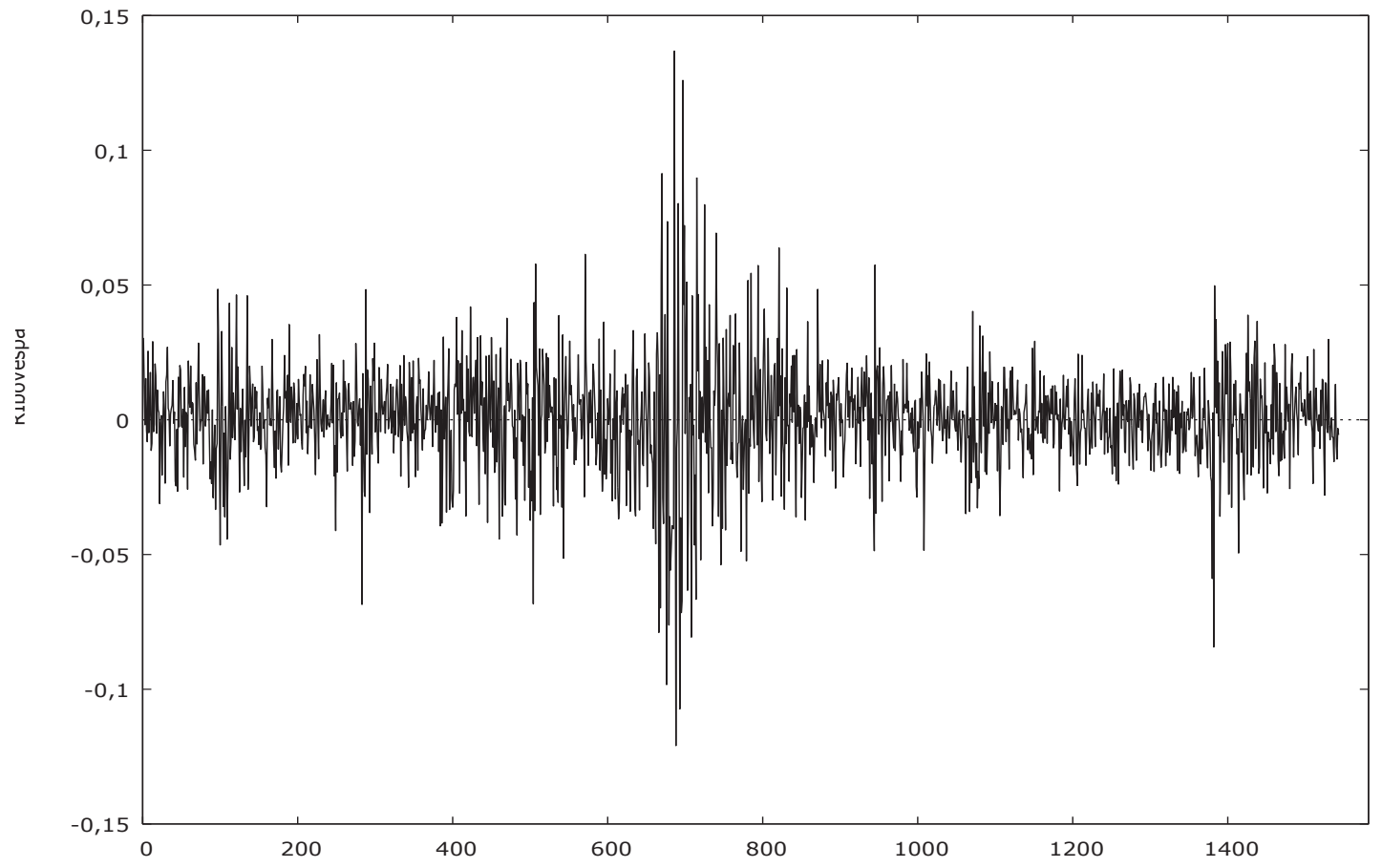

Figura 2 - Variação dos retornos dos índices de melhores práticas
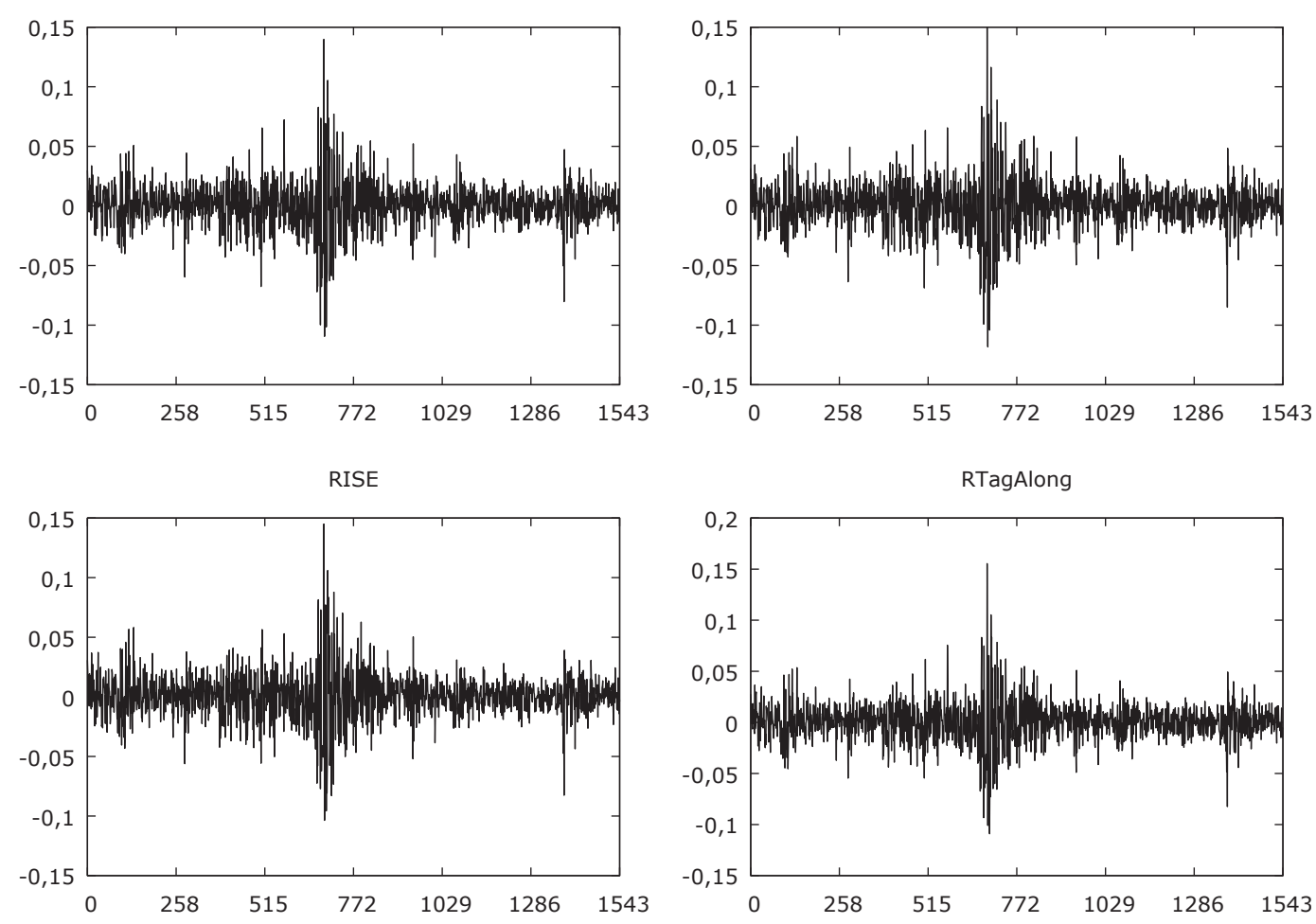

As Figuras 3 e 4 apresentam, respectivamente, a volatilidade do Ibovespa e a volatilidade dos índices de melhores práticas. 
Figura 3 - Volatilidade do índice Ibovespa

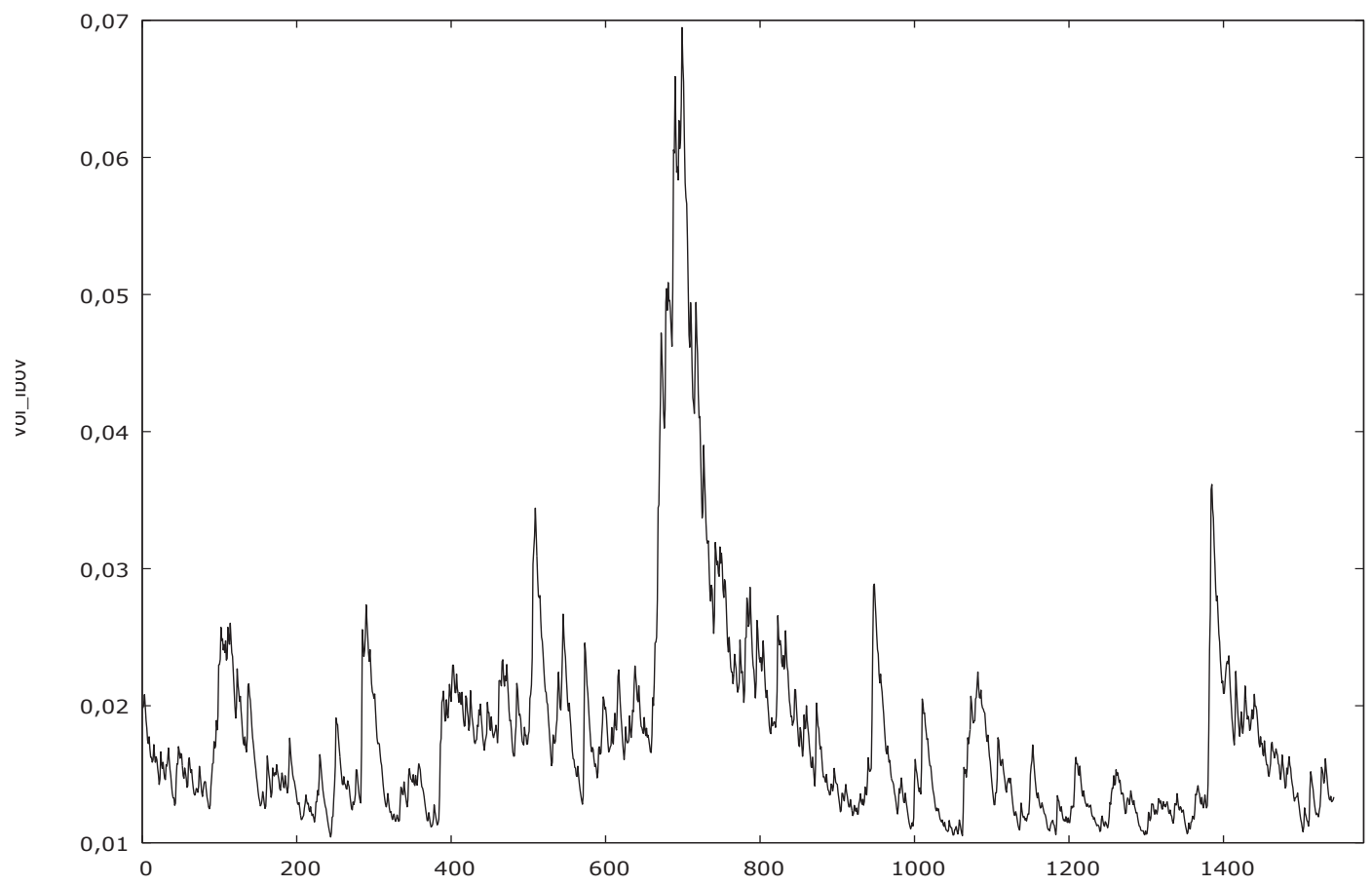

Figura 4 - Volatilidade dos índices de melhores práticas
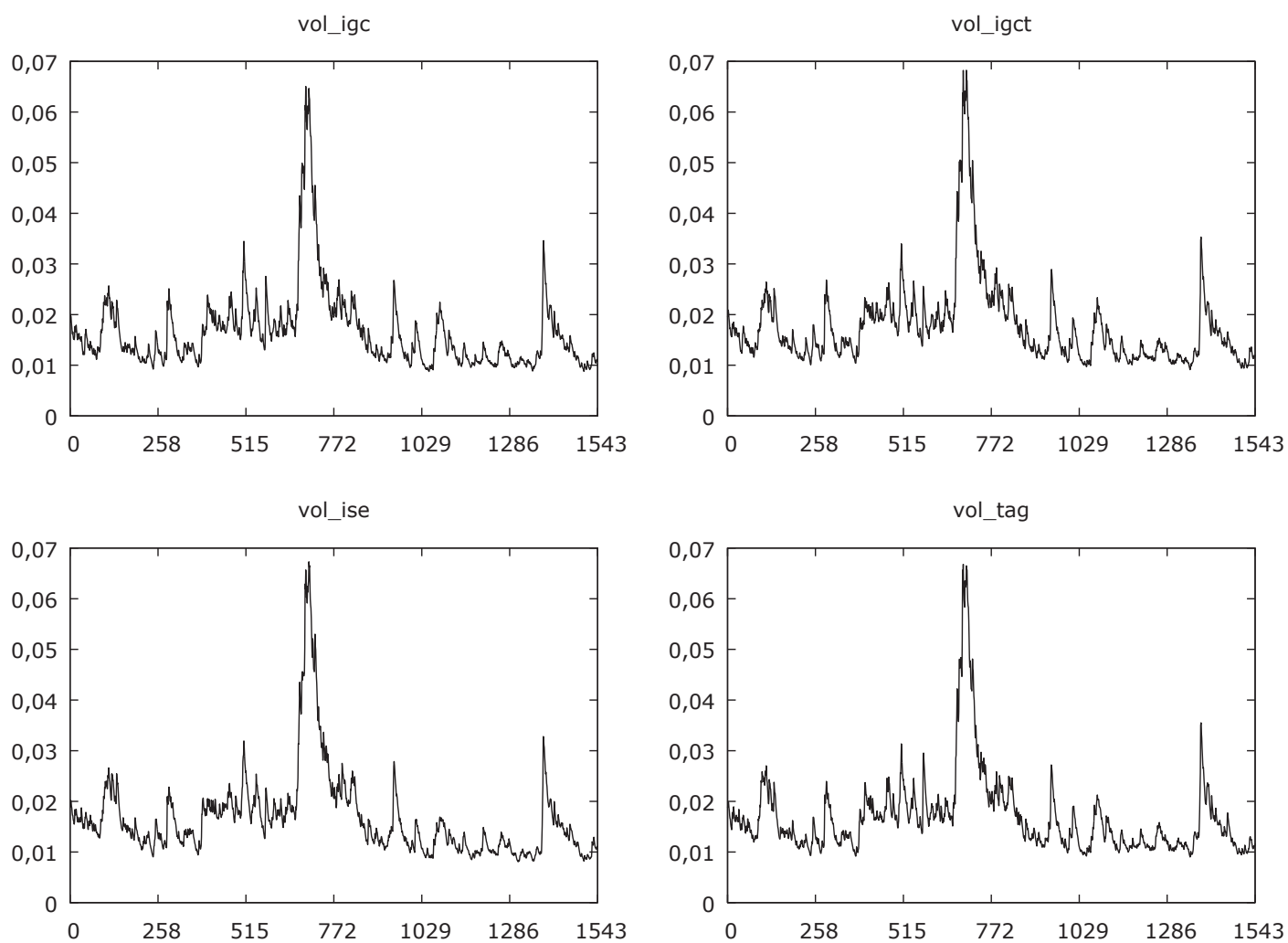
As Figuras 3 e 4 não apresentam nenhuma diferença de volatilidade entre os índices perceptível visual e graficamente, o que era esperado, tendo em vista que as Figuras 1 e 2 verificaram que a variação dos retornos para os mesmos índices é muito similar. Novamente, evidencia-se o aumento da volatilidade no período da crise do subprime, deixando claro que a volatilidade é heteroscedástica.

Para comparar a volatilidade condicional estimada para cada série, foi aplicado o teste de diferença de média de Wilcoxon (1945). Os p-valores do teste de diferença de média e a própria média de volatilidade estimada são apresentados na Tabela 6.

Tabela 6 - Média da volatilidade e p-valores do teste de diferença de rank

\begin{tabular}{ccccccc}
\hline \multirow{2}{*}{ Índice } & \multicolumn{3}{c}{ P-valor do teste de Wilcoxon } & Volatilidade \\
\cline { 2 - 5 } & Ibovespa & IGC & IGCT & ISE & ITAG & Média \\
\hline Ibovespa & 1,000 & 0,000 & 0,000 & 0,000 & 0,000 & 0,0180 \\
IGC & & 1,000 & 0,000 & 0,000 & 0,000 & 0,0166 \\
IGCT & & & 1,000 & 0,000 & 0,000 & 0,0176 \\
ISE & & & 1,000 & 0,000 & 0,0167 \\
ITAG & & & & 1,000 & 0,0169 \\
\hline
\end{tabular}

O teste de Wilcoxon (1945) rejeita a hipótese nula, ao grau de significância de 1\%, de que as volatilidades médias estimadas para cada índice sejam iguais. Ou seja, é possível afirmar que os índices não estão sujeitos à mesma volatilidade. Levando em consideração suas médias, percebe-se que o Ibovespa apresenta a maior volatilidade estimada; e o IGC, a menor. Como todos os demais índices obtiveram média de volatilidade condicional inferior ao lbovespa, pode-se dizer que os investimentos em empresas com melhores práticas são menos arriscados do que os investimentos nas empresas que representam o mercado.

Para analisar a performance dos índices em questão, foi calculada a média do Índice de Sharpe no período e o teste de diferença de média (Wilcoxon), para verificar se as médias diferem significativamente. A Tabela 7 apresenta a média do Índice de Sharpe (1966) para cada variável e o teste de diferença de média entre elas.

Tabela 7 - Índice de Sharpe dos Índices

\begin{tabular}{ccccccc}
\hline \multirow{2}{*}{ Índice } & \multicolumn{3}{c}{ P-valor do teste de Wilcoxon } & \multicolumn{2}{c}{ Índice de Shar- } \\
\cline { 2 - 5 } & Ibovespa & IGC & IGCT & ISE & ITAG & 0,0230 \\
\hline Ibovespa & 1 & 0,0000 & 0,0000 & 0,0000 & 0,0000 & 0,0369 \\
IGC & & 1,0000 & 0,0000 & 0,0000 & 0,0000 & 0,0332 \\
IGCT & & & 1,0000 & 0,0000 & 0,0000 & 0,0361 \\
ISE & & & 1,0000 & 0,0000 & 0,0358 \\
ITAG & & & & 1,0000 & 0 \\
\hline
\end{tabular}

Os dados apresentados na Tabela 7 demonstram que o teste de diferença de média rejeita a hipótese nula de que as médias sejam iguais, ao grau de significância de $1 \%$, em todas as relações. O Índice de Sharpe (1966) do Ibovespa foi consideravelmente inferior aos demais, deixando claro que esta foi a pior opção de investimento no período analisado. Por outro lado, o IGC apresentou a maior média, podendo ser considerado a melhor alternativa no período. Chama a atenção o fato de que os índices de melhores práticas apresentam valores de índice de Sharpe próximos entre si, mas o do lbovespa foi consideravelmente menor.

Portanto, os resultados indicam que, no período analisado, as empresas listadas nos índices de melhores práticas tiveram melhor performance do que as empresas listadas no Iboves- 
pa, que representa o mercado como um todo.

\section{CONSIDERAÇÕES FINAIS}

O presente estudo teve como objetivo mensurar a performance de diversos índices calculados pela BMF\&BOVESPA, a fim de verificar se há diferenças entre índices que listam empresas com melhores práticas de Sustentabilidade, Responsabilidade Social, Governança Corporativa e o lbovespa, que representa o mercado.

Com base nas estimações da volatilidade condicional, o teste de diferença de média de Wilcoxon (1945), foi verificado que o Ibovespa tem volatilidade significativamente superior aos demais índices ao grau de significância de 1\%, o que permite deduzir que o investidor que aplicar seus recursos numa carteira que visa a imitar o comportamento desse índice pode estar sujeito a um risco maior do que aquele que balizar seus investimentos nas empresas listadas nos índices de sustentabilidade.

A relação entre risco e retorno também foi analisada, tomando por base o Índice de Sharpe (1966) e a volatilidade condicional. Os resultados apontam que o índice Ibovespa apresenta a menor média de retorno por unidade de risco, o que foi comprovado pelo teste de diferença de média de Wilcoxon (1945). Assim, é possível afirmar que o Ibovespa apresentou a pior performance entre os índices analisados.

Cabe destacar que o IGC apresentou a menor volatilidade entre todos os índices, bem como a maior média de retorno por unidade de risco, o que permite afirmar que esse foi o índice com meIhor performance no período, superando o lbovespa e os demais índices de melhores práticas.

É possível concluir que os resultados deste estudo estão alinhados com Ortas, Moneva e Salvador (2010); Hoti, McAleer e Pauwels (2008); e Rabelo et. al (ANO) no sentido de que a volatilidade dos índices de melhores práticas é inferior. Levando-se em conta que os índices que representam as melhores práticas apresentam retorno por unidade de risco significativamente superior, é possível concluir que eles são benéficos também aos shareholders, além dos stakeholders. 


\section{REFERÊNCIAS}

BM\&FBOVESPA, disponível em www em 20/06/2012.

BOLLERSLEV, T. Generalized autoregressive conditional heteroskedacity. Journal of Econometrics, v. 53, p. 307-327, 1986.

BOWEN, H. Responsabilidade social do homem de negócios. Rio de Janeiro: Civilização, 1957

BUENO, R. L. S. Econometria de Séries Temporais. 1 reimpressão., 1 ed., São Paulo: Cengage Learning, 2011.

CARROLL, A. Corporate Social Responsibility Evolution of a Definitional Construct. Business \& Society, v. 38, n. 3, p. 268-295, 1999.

DIAS, Valéria da Veiga. Análise de Práticas de Gestão Sustentável em Empresas Internacionalizadas do Setor Químico Brasileiro. Dissertação (Mestrado em Administração) Programa de Pós-Graduação em Administração, UFSM, Santa Maria, 2011.

ENGLE, R.F. Autoregressive conditional Heteroskedacity with the estimates of variance of the United Kingdon inflation. Econometrica, v. 50, n. 4, p. 987-1008, 1982.

HOTI, S.; MCALEER, M.; PAUWELS, L.L. Multivariate Volatility in environmental finance. Mathematics and Computing in Simulation. v. 78, p. 189-199, 2008.

JUBERT, R. W.; MONTE, P. A. PAIXÃO, M. C. S.; LIMA, W. H. Um estudo do padrão de volatilidade dos principais índices financeiros do Bovespa: uma aplicação de modelos ARCH. UnB Contábil, v. 11, n. 1-2, p. 221-239, 2008.

LEFF, E. Educaçãoambientale desenvolvimento sustentável. Rio de Janeiro: DP\&A, 2001.

LJUNG, G. M.; BOX, G. E. P. The likelihood function of stationary autoregressive-moving average models. Biometrika, n. 66, v. 2, p. 265270, 1979.
MARKOWITZ, H. Portfolio Selection. The Journal of Finance, v. 7. n.1, 1952.

OLIVEIRA, Gilson Alves de; PACHECO, Marcelo Marques. Mercado Financeiro: Objetivo e Profissional. - São Paulo: Editora Fundamento Educacional, 2010.

ORTAS, E.; MONEVA, J.M.; SALVADOR, M. Conditional Volatility in Sustainable and traditional stock exchange indexes: analysis of the Spanish Market. Globalization, Competitiveness \& Governability, v. 4, n. 2, 2010.

RABELO, S. S. T.; ROGERS, P.; RIBEIRO, K. C. S.; SECURATO, J. R. Performance das Melhores Práticas de Governança Corporativa no Brasil: um estudo de carteiras. FACEF Pesquisa, v. 10, n. 2, 2007.

SCHWARTZ, M., CARROLL A. Frameworks: The search for a common core in the business and integrating and unifying competing and complementary society field Business and Society, v. 47, n. 2 , Sage Publications, 2008, p.148-186. Disponível em: http://bas.sagepub. com hosted at http://online.sagepub.com. Acesso dia: 05/11/2011.

SHARPE, William F. Mutual Fund Performance. The Journal of business, v. 39, n. 1, p.119-138, 1966.

WERRE, M. e VAN MARREWIJK, M. Multiple Levels of Corporate Sustainability. Journal of Business Ethics, v.44, p.107-119. 2003.

WILCOXON, Frank. Individual comparisons by ranking methods. Biometrics Bulletin n. 1, v. 6 , p. 80-83, 1945.

WILLARD, B. The Sustainability Advantage: Seven Business Case Benefits of a Triple Bottom Line. New Society Publishers. Gabriola Island, British Colombia: New Society, 2002 